\title{
Topical administration of DPP-IV inhibitors prevents retinal neurodegeneration in experimental diabetes
}

\author{
Cristina Hernández ${ }^{1,2} \cdot$ Patricia Bogdanov ${ }^{1,2} \cdot$ Cristina Solà-Adell $^{1,2} \cdot$ \\ Joel Sampedro ${ }^{1}$ Marta Valeri $^{3}$ - Xavier Genís ${ }^{4}$ - Olga Simó-Servat ${ }^{1,2}$. \\ Marta García-Ramírez ${ }^{1,2} \cdot$ Rafael Simó $^{1,2}$
}

Received: 2 February 2017 / Accepted: 21 June 2017 /Published online: 4 August 2017

(C) Springer-Verlag GmbH Germany 2017

\begin{abstract}
Aims/hypothesis The main aims of the present study were: (1) to assess the expression and content of dipeptidyl peptidase IV (DPP-IV) in human and $d b / d b$ mouse retinas, and in human vitreous fluid; and (2) to determine whether the topical administration of the DPP-IV inhibitors (DPP-IVi) would prevent retinal neurodegeneration and vascular leakage in $d b / d b$ mice by reducing endogenous glucagon-like peptide 1 (GLP-1) degradation.

Methods To assess the expression and content of DPP-IV, human samples of vitreous fluid and retinas were obtained from participants with type 2 diabetes $(n=8)$ and agematched non-diabetic individuals $(n=8)$, as well as from $d b / d b(n=72)$ and $d b /+(n=28)$ mice. The interventional study, which included $72 \mathrm{db} / \mathrm{db}$ mice, consisted of the topical administration (eye drops) of saxagliptin, sitagliptin or vehicle for 14 days. DPP-IV mRNA levels were assessed by RT-PCR,
\end{abstract}

Cristina Hernández and Patricia Bogdanov contributed equally to this study.

Electronic supplementary material The online version of this article (doi:10.1007/s00125-017-4388-y) contains peer-reviewed but unedited supplementary material, which is available to authorised users.

Rafael Simó

rafael.simo@vhir.org

1 Diabetes and Metabolism Research Unit, Vall d'Hebron Research Institute, Pg. Vall d'Hebron 119-129, 08035 Barcelona, Spain

2 Centro de Investigación Biomédica en Red de Diabetes y Enfermedades Metabólicas Asociadas (CIBERDEM), Instituto de Salud Carlos III (ISCIII), Madrid, Spain

3 Unit of High Technology, Vall d'Hebron Research Institute, Barcelona, Spain

4 Banco de Sangre y Tejidos, Passeig Taulat 116, 08005 Barcelona, Spain and protein content was measured by ELISA or western blotting. GLP-1 was assessed by immunofluorescence, and its downstream effector exchange protein activated by cAMP-1 (EPAC-1) was used as a measure of GLP-1 receptor activation. Retinal analyses were performed in vivo by electroretinography and ex vivo by RT-PCR (Epac-1, Iba-1 [also known as Aif1]), western blotting (EPAC-1, glial fibrillar acidic protein [GFAP], glutamate-aspartate transporter [GLAST]) and immunofluorescence measurements (GLP-1, GFAP, ionised calcium binding adaptor molecule 1 [IBA-1], TUNEL, GLAST, albumin and collagen IV). Glutamate was quantified by HPLC. In addition, vascular leakage was examined by the Evans Blue method.

Results DPP-IV was present in human vitreous fluid but in a range 100-fold less than in plasma. Both mRNA levels and protein content were much lower in the retina than in the liver or bowel, but were significantly higher in retinal pigment epithelium (RPE) from diabetic donors in comparison to non-diabetic donors $(p<0.05)$. Topical treatment with DPP-IVi prevented glial activation, apoptosis and vascular leakage induced by diabetes in $d b / d b$ mice $(p<0.05)$. Moreover, it also significantly prevented diabetes-induced functional abnormalities in the electroretinogram. A significant increase of both GLP-1 and EPAC-1 was found after treatment with DPP-IVi $(p<0.05)$. Furthermore, GLAST downregulation induced by diabetes was prevented, resulting in a significant reduction of extracellular glutamate concentrations. All these effects were observed without any changes in blood glucose levels.

Conclusions/interpretation The topical administration of DPP-IVi is effective in preventing neurodegeneration and vascular leakage in the diabetic retina. These effects can be attributed to an enhancement of GLP-1, but other mechanisms unrelated to the prevention of GLP-1 degradation cannot be ruled out. 
Keywords Diabetic retinopathy · DPP-IV inhibitors . Experimental diabetes $\cdot$ GLP-1 $\cdot$ Retinal neurodegeneration

$\begin{array}{ll}\text { Abbreviations } \\ \text { BRB } & \text { Blood-retinal barrier } \\ \text { DPP-IV } & \text { Dipeptidyl peptidase IV } \\ \text { DPP-IVi } & \text { Dipeptidyl peptidase IV inhibitor } \\ \text { EPAC-1 } & \text { Exchange protein activated by cAMP-1 } \\ \text { F2RL1 } & \text { Factor II receptor-like 1 } \\ \text { GFAP } & \text { Glial fibrillar acidic protein } \\ \text { GLAST } & \text { Glutamate-aspartate transporter } \\ \text { GLP-1 } & \text { Glucagon-like peptide 1 } \\ \text { GLP-1R } & \text { Glucagon-like peptide 1 receptor } \\ \text { IBA-1 } & \text { Ionised calcium binding adaptor molecule 1 } \\ \text { IL-1RA } & \text { Interleukin-1 receptor antagonist } \\ \text { RPE } & \text { Retinal pigment epithelium } \\ \text { STZ } & \text { Streptozotocin } \\ \text { SUSTAIN-6 } & \text { Cardiovascular and Other Long-term } \\ & \text { Outcomes with Semaglutide in Subjects } \\ & \text { with Type 2 Diabetes } \\ \text { VEGF } & \text { Vascular endothelial growth factor }\end{array}$

\section{Introduction}

Although diabetic retinopathy has been classically considered to be a microcirculatory disease of the retina, there is emerging evidence to suggest that retinal neurodegeneration is an early event in the pathogenesis of diabetic retinopathy that could participate in the development of microvascular abnormalities [1-6]. In fact, neural apoptosis and reactive gliosis, the hallmarks of retinal neurodegeneration, have already been observed in diabetic donors without microcirculatory abnormalities $[7,8]$. There are several possible approaches to treating neurodegeneration (i.e. blocking the glutamate signalling pathway, replacing downregulated neuroprotective factors and improving the neurovascular coupling function), which have been reviewed [1].

We have recently found that the glucagon-like peptide 1 (GLP-1) receptor (GLP-1R) is abundantly produced in the human retina, and that the topical administration of GLP-1R agonists is able to prevent the neurodegenerative process (reactive gliosis and apoptosis) in the retina in a mouse model of spontaneous type 2 diabetes ( $d b / d b$ mouse) that also expresses GLP-1R in the retina [9]. In addition, we found the presence of GLP-1 in the human retina, but both mRNA levels and protein content were significantly lower in retinas from diabetic in comparison with control individuals.

Furthermore, the effect of GLP-1 administered via eye drops was very similar to that of GLP-1R analogues. This was an unexpected finding because native GLP-1 is extremely susceptible to the catalytic activity of the enzyme dipeptidyl peptidase IV (DPP-IV), which cleaves off the two $\mathrm{NH}_{2}$-terminal amino acids. Consequently, GLP-1 rapidly degrades, showing a half-life in plasma of 1-2 min [10]. However, the presence of DPP-IV in the human retina and vitreous humour has never been examined. In addition, the potential capacity of DPP-IV inhibitors (DPP-IVi) administered by the topical route (i.e. as eye drops) in preventing retinal neurodegeneration induced by diabetes remains to be investigated.

On this basis, the aims of the present study were: (1) to assess the expression and content of DPP-IV in the human retina and vitreous fluid; (2) to determine whether the topical administration (eye drops) of the DPP-IVi saxagliptin and sitagliptin would reduce GLP-1 degradation, thus preventing neurodegeneration and vascular leakage in $d b / d b$ mice; and (3) to investigate whether, as occurs with GLP-1, the amelioration of glutamate-mediated excitotoxicity would be among the mechanisms involved in its potential beneficial effects.

\section{Methods}

\section{Human samples}

Vitreous fluid The study included eight participants with type 2 diabetes who had undergone vitrectomy owing to proliferative diabetic retinopathy. Eight age-matched non-diabetic participants with other conditions requiring vitrectomy (epiretinal membrane or macular hole) served as a control group. Undiluted vitreous samples were obtained at the onset of vitrectomy. Vitreous samples from individuals who had received intravitreal injections of anti-vascular endothelial growth factor (VEGF) agents or corticosteroids 6 months prior to vitrectomy were rejected.

All individuals included in the study were recruited from Vall d'Hebron University Hospital, Barcelona, Spain. The research followed the tenets of the Declaration of Helsinki. The protocol for sample collection was approved by the hospital ethics committee, and the study participants gave informed consent.

Retinas Retinas were obtained from the tissue bank of our centre (Banc de Sang i Teixits Hospital Universitari Vall d'Hebron). Eight diabetic and eight non-diabetic donors matched by age and sex were included in the study. Fundoscopic examination of diabetic donors performed the year before death revealed the absence of microvascular abnormalities or mild non-proliferative diabetic retinopathy. Accordingly, these selected retinas had not previously been treated with intravitreous corticosteroids or anti-VEGF injections. The general characteristics of participants with diabetes and non-diabetic control individuals, the cause of death and 
glucose-lowering treatment are summarised in electronic supplementary material [ESM] Table 1.

One eye-cup was harvested in order to separate the neuroretina from the retinal pigment epithelium (RPE), and both tissues were immediately frozen with liquid nitrogen and stored at $-80^{\circ} \mathrm{C}$. Tissues derived from this eye-cup were used for the studies of gene expression and protein measurement. The other eye-cup was also harvested, and both the RPE and neuroretina were soaked in paraffin and used for immunohistochemical studies. The time period from death to eye enucleation was $3.7 \pm 1.5 \mathrm{~h}$

The procedure for eye-cup donation and for the handling of this biological material was regulated by the protocol of donations of the tissue bank of our centre and was approved by the ethical committee.

RNA extraction and quantitative RT-PCR The relative expression levels of mRNA were quantified by quantitative RTPCR (see ESM Methods). The primers used are detailed in ESM Table 2.

Immunohistochemistry Retinal sections from the eight diabetic and eight non-diabetic donors were deparaffinised in xylol and were rehydrated in graded ethanol. To eliminate autofluorescence, slides were washed in potassium permanganate. The methodology used is detailed in the ESM Methods.

ELISA DPP-IV was assessed in plasma, vitreous and retinal extracts (RPE and neuroretina) by a quantitative sandwich enzyme immunoassay (R\&D Systems, Minneapolis, MN, USA) with a lower limit of detection of $0.016 \mathrm{ng} / \mathrm{ml}$. The DPP-IV concentration in vitreous fluid and retinal extracts was normalised to total protein levels. Total protein concentration in the retinal and vitreous samples was obtained by bicinchoninic acid (BCA) assay (Thermo Scientific, Rockford, IL, USA).

\section{Animal studies}

Experimental design Two separate studies were performed, one testing saxagliptin and the other testing sitagliptin. A total of 36 male $d b / d b$ (BKS.Cg-Dock $7 \mathrm{~m}^{+/+}$Leprdb/J) mice aged 8 weeks were purchased (Charles River Laboratories, Calco, Italy) for the saxagliptin study. In addition, 14 non-diabetic $\left(\mathrm{db} /+\right.$; BKS.Cg-Dock $7 \mathrm{~m}^{+/+}$Leprdb/+) mice matched by age were used as a control group.

Animals were acclimated 2 weeks prior to the study under tight environmental conditions of humidity (60\%), temperature $\left(20^{\circ} \mathrm{C}\right)$ and cycles of $12 \mathrm{~h} / 12 \mathrm{~h}$ light/darkness. They had free access to filtered water and ad libitum food (ENVIGO Global Diet Complete Feed for Rodents, Mucedola, Milan, Italy). Then, in order to minimize variability, animals were randomly housed (block randomisation) in groups of four mice per cage. Each cage contained nesting material and absorbent bedding (BioFresh Performance Bedding 1/8" Pelleted Cellulose, Absorption Corp, Ferndale, WA, USA).

When the mice were aged 10 weeks, saxagliptin or vehicle eye drops were administered directly onto the superior corneal surface of each eye using a syringe. One drop $(5 \mu \mathrm{l})$ of saxagliptin $(1 \mathrm{mmol} / \mathrm{l} ; 31.5 \mathrm{mg} / \mathrm{ml})$, or vehicle $(5 \mu \mathrm{PBS}$, $\mathrm{pH}$ 7.4) was administered twice daily for 14 days. On day 15 , one drop of either saxagliptin or vehicle was administered to the animals' eyes $1 \mathrm{~h}$ prior to euthanasia.

The experimental design used for sitagliptin was the same as for saxagliptin, and a further 36 male $d b / d b$ mice and 14 $d b /+$ mice were used (supplier details the same). For the sitagliptin study, the concentration in the eye drops was $50 \mathrm{mg} / \mathrm{ml}$ sitagliptin.

On day 15 , the mice were anaesthetised (1 ml ketamine/ $0.3 \mathrm{ml}$ xylazine) and transcardially perfused. The animals were euthanised by cervical dislocation, and the eyes were immediately enucleated. This study was approved by the Animal Care and Use Committee of VHIR (Vall d'Hebron Research Institute). All the experiments were performed in accordance with the tenets of the European Community (86/ 609/CEE) and the Association for Research in Vision and Ophthalmology (ARVO).

Electroretinograms Full-field electroretinogram recordings were measured using the Ganzfeld ERG platform (Phoenix Research Laboratories, Pleasanton, CA, USA), as reported elsewhere [11] and following the recommendations of the International Society for Clinical Electrophysiology of Vision [12].

Tissue processing The eyes were immediately enucleated, and the neuroretinas were separated (see ESM Methods). For immunohistochemical analysis, five sections of retina were obtained per animal. Images were acquired with a confocal laser scanning microscope (FV1000; Olympus, Hamburg, Germany). Five fields (three corresponding to the central and two to the peripheral retina) from each section were analysed. The same locations and number of fields were measured in all retinas.

RNA isolation and quantitative RT-PCR assay Quantitative RT-PCR was used to determine the relative expression levels of mRNAs (see ESM Methods). The primer sequences used in this study are shown in ESM Table 2.

Western blotting Proteins were extracted from the neuroretinas in $80 \mu \mathrm{l}$ of lysis buffer (RIPA buffer: phenylmethanesulfonylfluoride [PMSF], $1 \mathrm{mmol} / \mathrm{l} ; \mathrm{Na}_{3} \mathrm{VO}_{4}$, $2 \mathrm{mmol} / \mathrm{l}$; NaF, $100 \mathrm{mmol} / \mathrm{l}$ ) and $1 \mathrm{X}$ protease inhibitor cocktail (Sigma, St Louis, MO, USA) (see ESM Methods). 
Immunohistochemical analysis for glial activation assessment Glial activation was evaluated by fluorescence microscopy using specific antibodies against glial fibrillar acidic protein (GFAP) following the methodology described elsewhere [13]. To evaluate the degree of glial activation, we used a scoring system based on the extent of GFAP staining [14] that has previously been used by our group $[9,11,13]$.

Immunohistochemical analysis for apoptosis assessment Apoptosis was evaluated using the TUNEL method, as previously described [13] (see ESM Methods). The results are presented as the percentage of TUNEL-positive cells with respect to the Hoechst-stained cells obtained by Image $\mathrm{J}$ software (National Institutes of Health, Bethesda, MD, USA).

Measurement of retinal vascular permeability Retinal vascular permeability was examined by assessing albumin leakage from the blood vessels into the retina using doubleimmunostaining for albumin and collagen IV (in vitro) and the Evans Blue albumin method (ex vivo).

In the process of double-immunostaining for albumin and collagen IV, labelling with collagen IV localised the basement membrane of vessels and allowed us to evaluate the integrity of the blood-retinal barrier (BRB) by examining whether the albumin molecules remain confined within the retinal vessels or were in contrast extravasated (see ESM Methods).

The Evans Blue albumin method was used as previously described with some modifications [14-17] (see ESM Methods). Digital images from different random fields of all retinas were acquired using a confocal laser scanning microscope (FV1000; Olympus) at $\times 20$ using the $561 \mathrm{~nm}$ laser line, and each image was recorded with identical beam intensity at a size of 1024 pixels $\times 1024$ pixels. For quantitative analysis of the albumin-bound Evans Blue, Z-stacks retinal images (step size $1.16 \mu \mathrm{m}$ ) of different regions of the vascular tree were acquired. To remove the eye artefacts caused by the sample procedures, projections of the middle Z-plane images were analysed with the magic wand tool (tolerance 120, eight pixels connected) of Fiji software (https://fiji.sc [18]).

Other immunohistochemistry analyses GLP-1 and glutamate-aspartate transporter (GLAST) were evaluated by fluorescence microscopy using specific antibodies, as previously reported [9].

Glutamate quantification The quantification of glutamate was performed by reverse-phase ultraperformance liquid chromatography (Acquity UPLC; Waters, Milford, MA, USA) as aminoquinoline derivatives (AccQ-Tag Chemistry, MassTrak AAA method and instruments; Waters), following the methodology previously described by Narayan et al [19].

\section{Statistical analysis}

Data are presented as means $\pm \mathrm{SD}$. Comparisons of continuous variables were performed using ANOVA and Student's $t$ test with SPSS software (SPSS/Windows version 18; SPSS, Chicago, IL, USA). The statistical significance level was set at $p<0.05$.

\section{Results}

\section{DPP-IV mRNA levels and protein concentration} in the human retina

DPP-IV was expressed in the human retina but at significantly lower mRNA levels than in the liver and bowel. Nevertheless, a higher expression of DPP-4 mRNA was detected in RPE from diabetic donors in comparison with non-diabetic donors $(5.69 \pm 1.77$ vs control $1.40 \pm 1.38 ; p<0.05)$. In contrast, in the neuroretina, no significant differences were observed between groups $(1.66 \pm 0.77$ vs $3.11 \pm 2.25 ; \mathrm{p}=\mathrm{NS})$ (Fig. 1a). Similarly, DPP-IV protein levels were significantly higher in RPE from diabetic donors than from non-diabetic donors $(p<0.05)$ (Fig. 1b). In contrast, no significant differences were observed in the neuroretina.

\section{DPP-IV concentrations in human vitreous fluid}

DPP-IV was also detected in the vitreous fluid but in a range 100 -fold less than in plasma. No differences in either plasma or intravitreal DPP-IV levels were observed between type 2 diabetic and non-diabetic participants (plasma $497 \pm 133 \mathrm{ng} / \mathrm{ml}$ vs $491 \pm 151 \mathrm{ng} / \mathrm{ml}$, respectively, $\mathrm{p}=\mathrm{NS}$; vitreous fluid $6.2 \pm 8.32 \mathrm{ng} / \mathrm{ml}$ vs $5.9 \pm 6.28 \mathrm{ng} / \mathrm{ml}$, respectively, $\mathrm{p}=\mathrm{NS}$ ).

\section{DPP4 mRNA levels and protein concentration in $d b / d b$ mouse retina}

As in the human samples, we did not find any significant differences in the mRNA and protein levels of DPP-IV between the neuroretinas from diabetic and non-diabetic mice (Fig. 1c, d). We were unable to obtain accurate data regarding the RPE because of difficulty in separating it from the choroid. In addition, the limited volume of vitreous fluid that can be obtained from $d b / d b$ mice prevented the measurement of DPP-IV levels.

\section{Topical administration of DPP-IVi increases the ocular content of GLP-1 and its downstream target EPAC-1}

Topical administration of both saxagliptin and sitagliptin induced an increase in the retinal content of GLP-1 (Fig. 2a, b). In addition, both DPP-IVis were able to significantly increase 
a

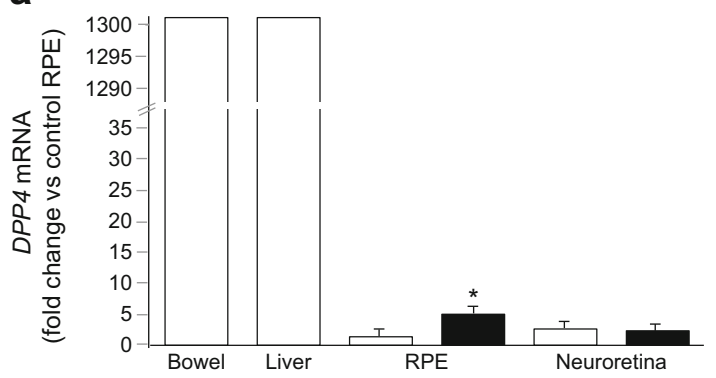

b

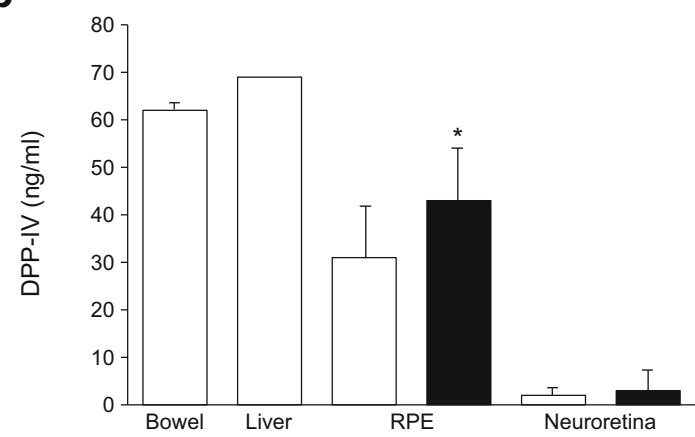

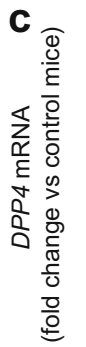

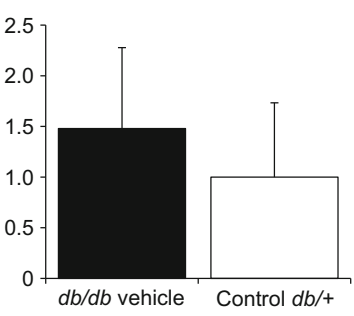

d

Fig. 1 (a) Real-time quantitative RT-PCR analysis of DPP4 mRNA and (b) DPP-IV concentration $(\mathrm{ng} / \mathrm{ml})$ in human retinas from diabetic $(n=8$; black bars) and non-diabetic ( $n=8$; white bars) donors; $* p<0.05$ between diabetic and non-diabetic donors. (c) Real-time quantitative RTPCR analysis of Dpp 4 mRNA in mouse retinas expressed as the fold change relative to control $(n=8)$. (d) Western blotting bands of DPP$\mathrm{IV}$ in mouse retina $(n=4)$. AU, arbitrary units

the levels of mRNA (Fig. 2c) and protein (Fig. 2d) for exchange protein activated by cAMP-1 (EPAC-1). This protein is a downstream cAMP signalling mediator and plays an important role in the maintenance of the endothelial barrier and neuronal functions [20-23].

\section{Neurodegeneration was prevented in diabetic mice treated with DPP-IVi}

Glial activation As expected, in non-diabetic mice, GFAP production was confined to the retinal ganglion cell layer, and the GFAP score was $\leq 2$ (Fig. 3). The diabetic mice treated with vehicle presented significantly higher GFAP levels than non-diabetic mice matched by age. Thus, all the diabetic mice presented a GFAP score $\geq 3$. Topical administration (eye drops) of either saxagliptin or sitagliptin for 2 weeks led to a significant decrease of reactive gliosis, and the GFAP score was $<3$ in all cases (Fig. 3). These results were confirmed by western blotting. In addition, a significant increase in ionised calcium binding adaptor molecule 1 (IBA-1) (mRNA and protein levels) was observed in diabetic mice, and this was preventable by the topical administration of DPP-IVi (Fig. 4).

Retinal apoptosis The apoptosis rate was significantly higher in diabetic mice treated with vehicle than in non-diabetic mice in all retinal layers (Fig. 5). The topical administration (eye drops) of either saxagliptin or sitagliptin for 2 weeks resulted in a significant prevention of apoptosis in all retinal layers (Fig. 5).

Topical administration of DPP-IVi prevents the increase of glutamate induced by diabetes by inhibiting GLAST downregulation

Glutamate levels ( $\mu \mathrm{mol} / \mathrm{g}$ protein) in the diabetic retinas were higher than in the non- diabetic retinas (Fig. 6a, b). In diabetic mice treated with saxagliptin or sitagliptin, glutamate concentrations were significantly lower than in diabetic mice treated with vehicle $(p<0.05)$ and similar to those of control mice $(\mathrm{p}=$ NS) (Fig. 6a, b).

Furthermore, we observed that the GLAST, the main glutamate transporter produced by Müller cells, was significantly decreased in the retinas of diabetic mice treated with vehicle in comparison with non-diabetic mice (Fig. 6c, d). In diabetic mice treated with saxagliptin or sitagliptin, this downregulation of GLAST induced by diabetes was prevented (Fig. 6c, d).

\section{Topical treatment with DPP-IVi prevents} electroretinogram abnormalities induced by diabetes

Topical treatment with saxagliptin or sitagliptin prevented the reduction of a-wave and b-wave amplitude induced by diabetes. An example of an electroretinogram in response to low, medium and high stimulus intensities in a representative nondiabetic mouse, a $d b / d b$ mouse treated with vehicle and a $d b /$ $d b$ mouse treated with saxagliptin or sitagliptin is shown in Fig. 7a, c. Quantitative measurements with statistical evaluation are shown in Fig. 7b, d.

\section{Topical administration of DPP-IVi prevents albumin leakage induced by diabetes}

The double-immunostaining for collagen IV and albumin allowed us to identify the extravascular location of albumin in $d b / d b$ mice treated with vehicle in comparison to control animals, thus revealing the disruption of the BRB. Sitagliptin and saxagliptin were able to prevent the extravasation of albumin (Fig. 8a). In addition, the beneficial effect of the topical treatment with sitagliptin on vascular leakage was clearly shown 

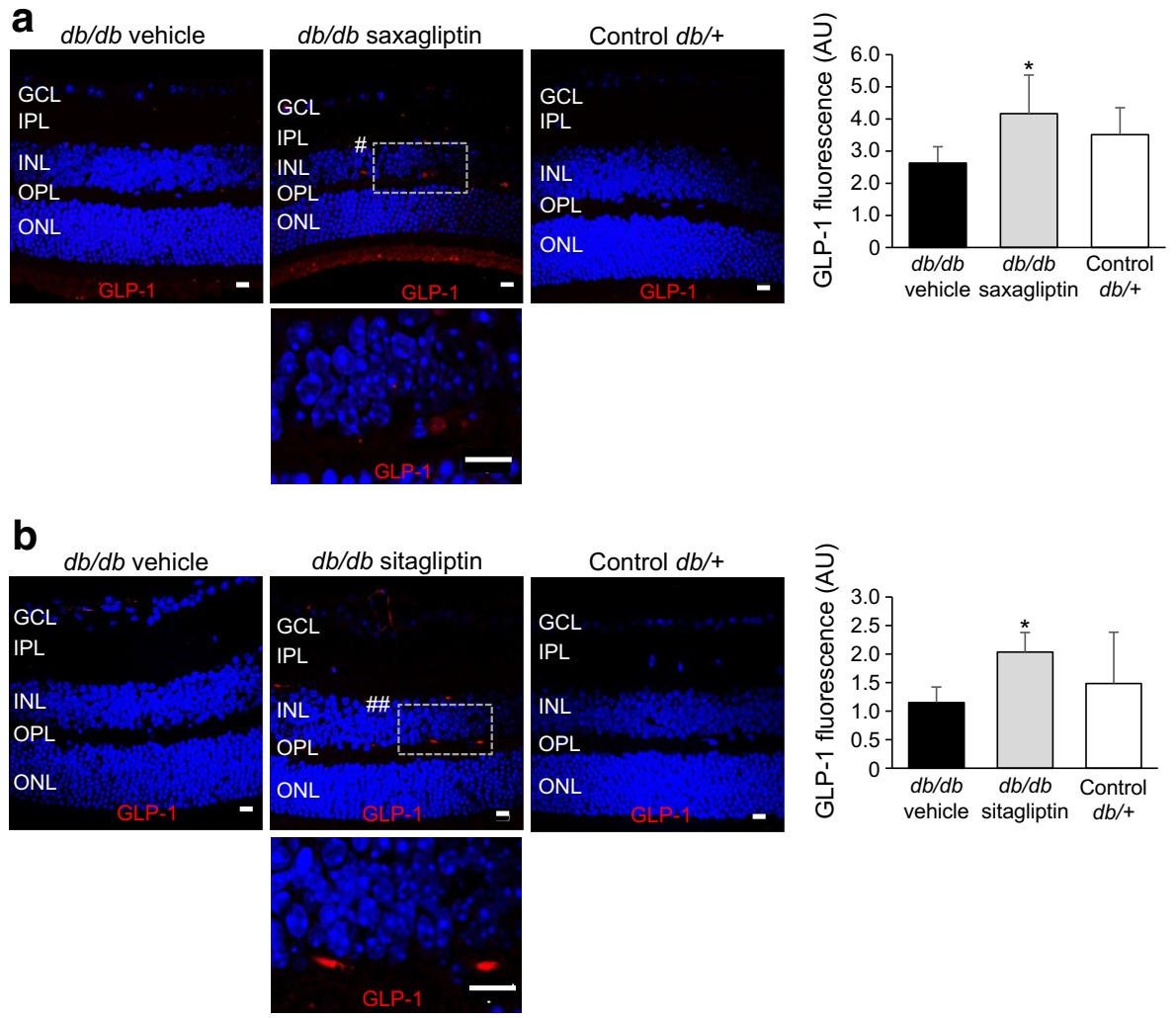

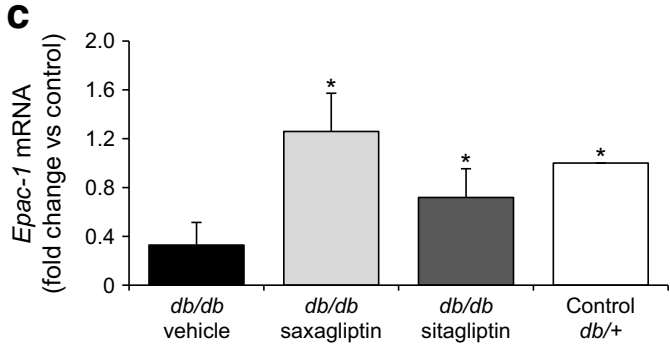

Fig. 2 (a, b) Immunofluorescence showing the increase in GLP-1 (red) in the retina after treatment with saxagliptin (a) and sitagliptin (b). Magnifications are shown of the areas within the dotted borders. Nuclei were labelled with Hoechst stain (blue). Scale bars, $10 \mu \mathrm{m}$. The graphs on the right show the fluorescence quantification. Data are expressed as means $\pm \mathrm{SD} ; n=10$ mice per group. (c) Real-time quantitative RT-PCR

using the Evans Blue method (Fig. 8b). Magnification of the images showing the albumin extravasation and the effect of the selected DPP-IVi in more detail is provided in ESM Fig. 1.

\section{The beneficial effects of the topical administration of DPP-IVi were unrelated to blood glucose levels}

Blood glucose concentrations and body weight at the end of treatment were similar in the $d b / d b$ mice treated with eye drops containing saxagliptin or sitagliptin and in the $d b / d b$ mice treated with eye drops containing vehicle (Tables 1 and 2). Therefore, the effects of the DPP-IVi were directly due to local effects in the retina and unrelated to the improvement of the diabetic milieu.

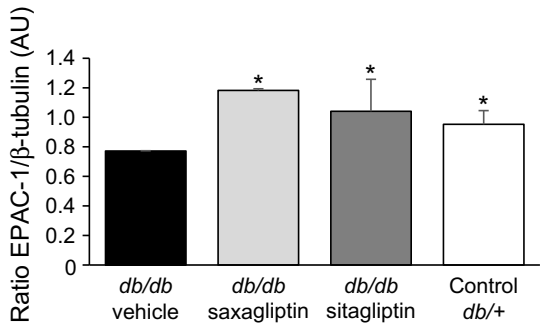

analysis of Epac-1 mRNA in mouse retinas. $n=8$. (d) Western blotting bands and quantification of EPAC-1 in mouse retina. $n=4$. $* p<0.05$ vs $d b / d b$ mice treated with vehicle. AU, arbitrary units; GCL, ganglion cell layer; INL, inner nuclear layer; IPL, inner plexiform layer; ONL, outer nuclear layer; OPL, outer plexiform layer

\section{Discussion}

In the present study, we have shown that both mRNA levels and protein content of DPP-IV are much lower in the retina than in the liver or bowel. Notably, DPP-IV levels in human vitreous fluid were around 100-fold lower than in plasma. These results could explain our previous observation regarding the capacity of GLP-1 to reach the retina after its topical administration and its neuroprotective actions through GLP1R. However, the higher DPP-IV concentrations detected in the RPE of diabetic in comparison with non-diabetic participants could decrease the availability of GLP-1 for reaching the neuroretina. In fact, the drugs that reach the retina via the transscleral route, as is the case for GLP-1, are first challenged 
Fig. 3 Glial activation. (a) Comparison of GFAP

immunoreactivity (green) among representative samples from diabetic mice treated with vehicle or saxagliptin and from a nondiabetic mouse. Scale bars, $20 \mu \mathrm{m}$. Quantification of glial activation based on the extent of GFAP staining. $n=10$ mice per group (five retinal sections per mouse). (b) Western blotting bands and quantification of GFAP in mouse retinas. $n=4$. ${ }^{*} p<0.05$ in comparison with the other groups. (c) Comparison of GFAP immunoreactivity (green) in the retina of representative samples from diabetic mice treated with vehicle or sitagliptin and from a non-diabetic mouse. Scale bars, $20 \mu \mathrm{m}$. Quantification of glial activation based on the extent of GFAP staining. $n=10$ mice per group (five retinal sections per mouse). (d) Western blotting bands and quantification of GFAP in mouse retinas. $n=4 . * p<0.05$ vs $d b / d b$ mice treated with vehicle. AU, arbitrary units; Cyp, ciclophyline; GCL, ganglion cell layer; INL, inner nuclear layer; IPL, inner plexiform layer; ONL, outer nuclear layer; OPL, outer plexiform layer. Nuclei were labelled with Hoechst stain (blue) a

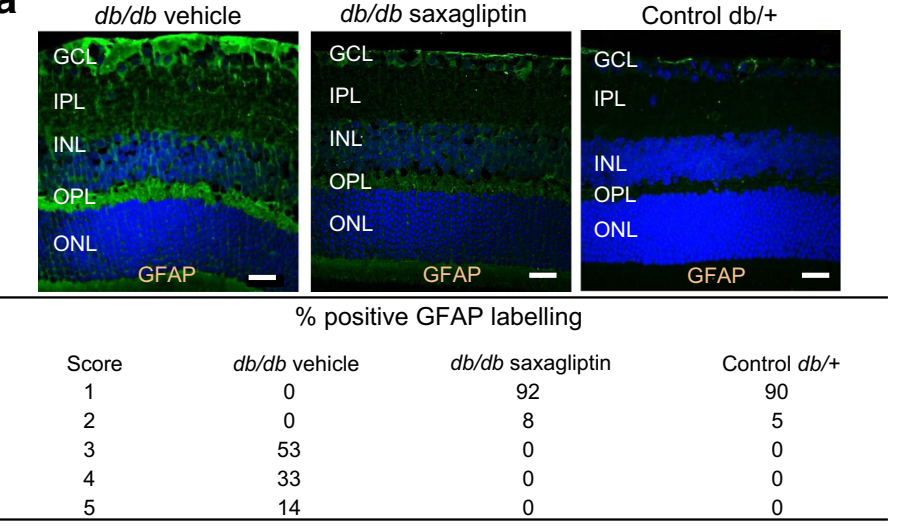

b
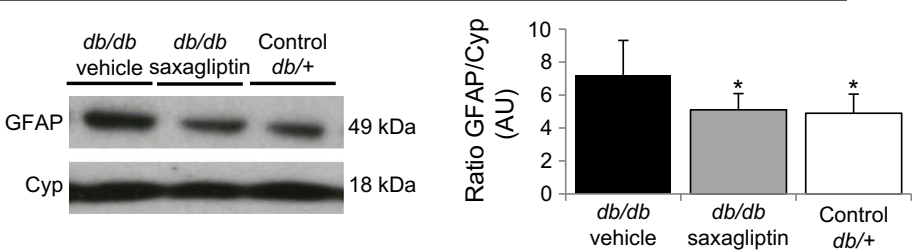

C

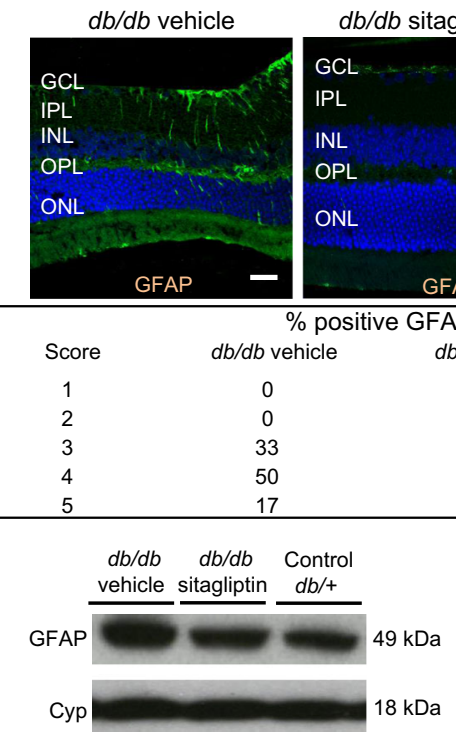

Control $d b /+$
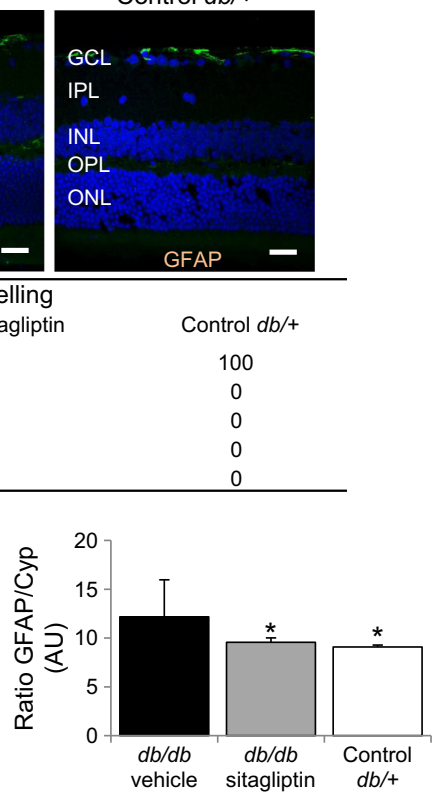

by the choroids and the RPE [24]. Therefore, a therapeutic strategy combining the topical administration of GLP-1 and a DPP-IVi could be a new and more effective approach to treating diabetic retinopathy.

From the pathophysiological point of view, it should be noted that GLP-1 is synthesised by the retina and its production is lower in diabetic individuals [9]. The local production of GLP-1 could be extremely important because of its potential autocrine and paracrine neuroprotective actions through GLP-1R. Therefore, the endogenous deficit of GLP-1 that exists in the diabetic retina could be involved in the development of neurodegeneration. Apart from its endogenous retinal source, GLP-1 could reach the retina from the systemic circulation by crossing the BRB.
At present, the proportion of locally derived and bloodborne GLP-1 that exists in the retina is unknown. Nevertheless, it seems reasonable to postulate that the preservation or enhancement of the retinal content of GLP-1 by preventing its degradation could be a new strategy for treating the early stages of diabetic retinopathy. The design of experiments addressed to test this hypothesis was the second aim of the present study, and we found that both DPP-IVi (saxagliptin and sitagliptin) administered as eye drops led to a significant increase in the intraretinal content of GLP-1, thus preventing neurodegeneration and vascular leakage in $d b / d b$ mice.

The mechanisms by which GLP-1R activation exerts its neuroprotective and vasculotropic action are complex and have recently been revised [25]. In the present study, we found 
Fig. 4 (a) Comparison of IBA-1 immunoreactivity (red) among representative samples from diabetic mice treated with vehicle, saxagliptin or sitagliptin and from a non-diabetic mouse; magnifications of images are shown below. Nuclei were labelled with Hoechst stain (blue). Scale bars, $10 \mu \mathrm{m}$. The graph shows quantification of IBA-1 staining. $n=10$ mice per group (five retinal sections per mouse). $* p<0.05$ in comparison with the other groups. (b) Real-time quantitative RT-PCR analysis of Iba-1 mRNA in mouse retinas. $n=8$. * $p<0.05$ vs $d b / d b$ mice treated with vehicle. GCL, ganglion cell layer; INL, inner nuclear layer; IPL, inner plexiform layer; ONL, outer nuclear layer; OPL, outer plexiform layer
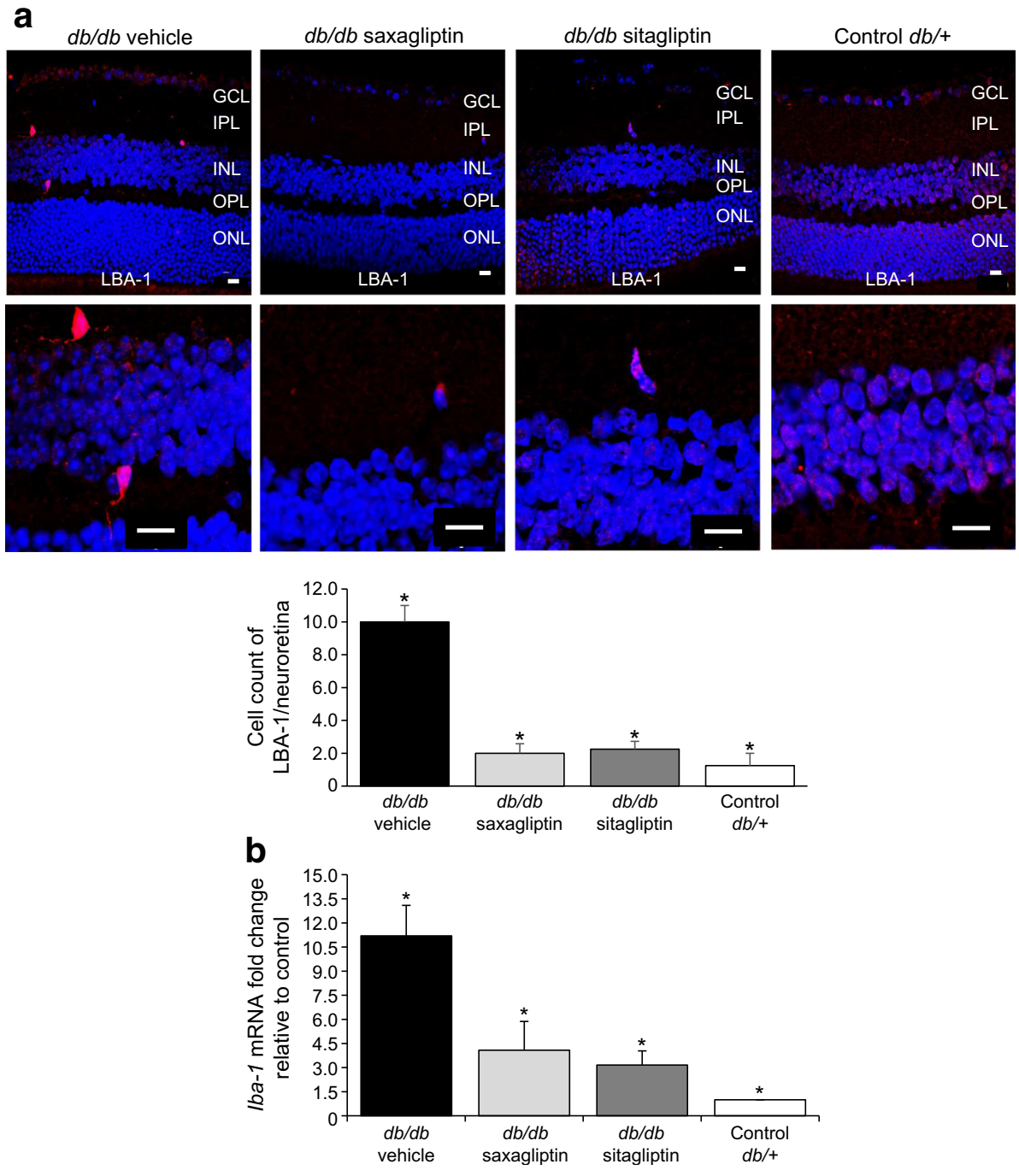

that saxagliptin and sitagliptin were able to increase both mRNA and protein levels of EPAC-1, a downstream cAMP signalling mediator. As cAMP is the main downstream messenger of GLP-1, it can be deduced that the EPAC-1 enhancement was due to GLP-1R activation. Interestingly, EPAC-1 plays an important role in the maintenance of the endothelial barrier and neuronal functions [20-23], and therefore its upregulation after DPP-IV inhibition indicates this pathway as a new mechanism contributing to the protective vasculotropic actions of GLP-1R activation.

However, the beneficial effects of saxagliptin and sitagliptin should not be exclusively attributed to an increase in GLP-1R activation. In fact, DPP-IVis themselves could activate unrelated downstream GLP-1R pathways that might be involved in neuroprotection. In this regard, Dietrich et al [26] recently reported that the DPP-IVi linagliptin has a neuroprotective effect in Caenorhabditis elegans, a model of neurodegeneration induced by high glucose levels in which GLP$1 \mathrm{R}$ is not produced. Experiments performed using experimental knockout models for GLP-1R would help us to elucidate this important issue. Nevertheless, our results suggest that preservation of the retinal content of GLP-1 (locally produced and serum derived) via topical administration of DPP-IVi seems a good strategy for treating diabetic retinopathy.

We used a topical rather than oral administration of DPPIVis in order to avoid the confounding factor represented by their capacity to lower blood glucose levels when they are orally administered. As we have shown in the present study, topical administration of either saxagliptin or sitagliptin was unable to change blood glucose levels, and consequently their effects were not mediated by an improvement of the diabetic milieu. With this strategy, we have been able to ascertain the direct effect of DPP-IVi independently of the improvement in blood glucose levels. As occurred with GLP-1R agonists [9], the topical administration of DPP-IVi also prevented the downregulation of GLAST induced by diabetes, thus reducing the extracellular concentration of glutamate, and consequently ameliorating excitotoxicity and retinal neuron death. In addition, the prevention of functional impairment measured by electroretinography was very similar to that previously 
Fig. 5 Apoptosis. (a) TUNELpositive immunofluorescence (green) in a representative $d b / d b$ mouse treated with vehicle, a $d b /$ $d b$ mouse treated with saxagliptin and a non-diabetic mouse. Scale bars, $10 \mu \mathrm{m}$. The table shows the percentage of TUNEL-positive cells in the neuroretina. Results are means \pm SD. $n=10$ mice per group. (b) TUNEL-positive immunofluorescence (green) in a representative $d b / d b$ mouse treated with vehicle, a $d b / d b$ mouse treated with sitagliptin and a non-diabetic mouse. Scale bars, $10 \mu \mathrm{m}$. The table shows the percentage of TUNEL-positive cells in the neuroretina. Results are means \pm SD. $n=10$ mice per group. $* p<0.05$ vs $d b / d b$ mice treated with vehicle. GCL, ganglion cell layer; INL, inner nuclear layer; IPL, inner plexiform layer; ONL, outer nuclear layer; OPL, outer plexiform layer. Nuclei were labelled with Hoechst stain (blue) a
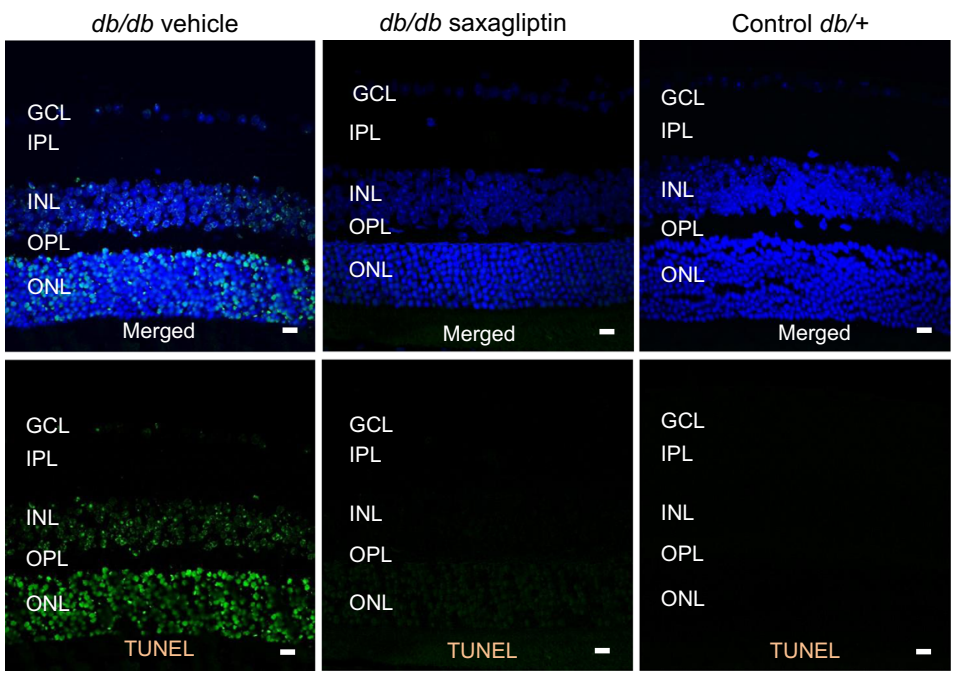

\begin{tabular}{clll}
\hline$\%$ TUNEL positive cells & $d b / d b$ vehicle & $d b / d b$ saxagliptin & Control $d b /+$ \\
GCL & $3.63 \pm 2.17$ & $0.04 \pm 0.01^{*}$ & $0.25 \pm 0.02^{*}$ \\
INL & $12.77 \pm 15.72$ & $0.86 \pm 1.07^{*}$ & $0.65 \pm 0.04^{*}$ \\
ONL & $28.09 \pm 6.66$ & $11.39 \pm 9.36^{*}$ & $1.24 \pm 0.14^{*}$ \\
\hline
\end{tabular}

b
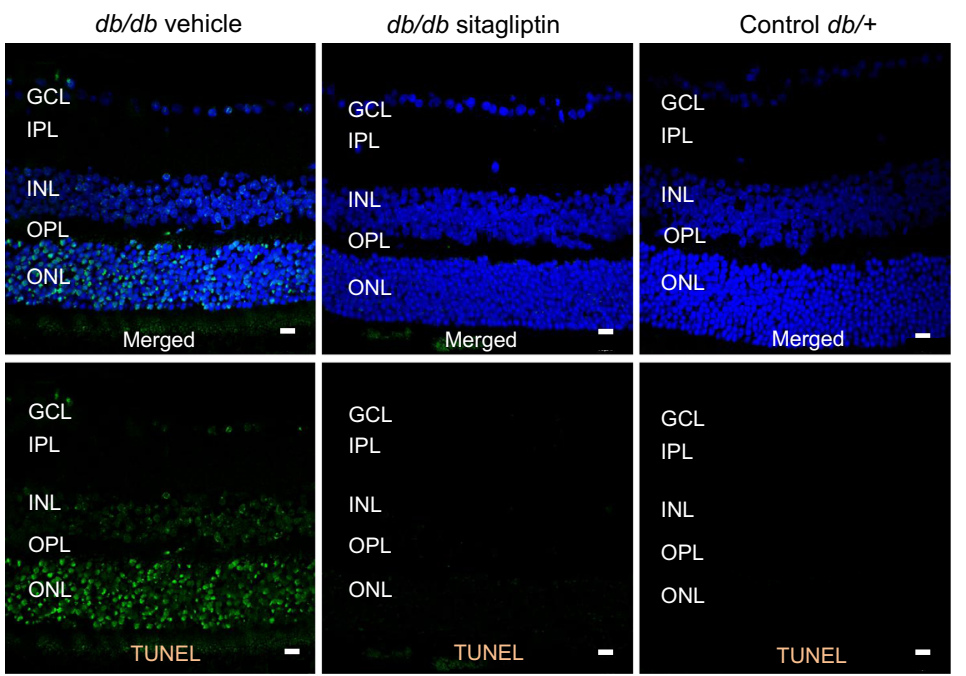

\begin{tabular}{clll}
\hline \% TUNEL positive cells & $d b / d b$ vehicle & $d b / d b$ sitagliptin & Control $d b /+$ \\
GCL & $4.87 \pm 0.89$ & $2.75 \pm 0.88^{*}$ & $1.00 \pm 0.71^{*}$ \\
INL & $15.50 \pm 4.03$ & $4.00 \pm 1.42^{*}$ & $1.00 \pm 0.71^{*}$ \\
ONL & $30.62 \pm 8.39$ & $5.12 \pm 5.12^{*}$ & $3.25 \pm 1.11^{*}$ \\
\hline
\end{tabular}

reported with GLP-1 [9]. Taken together, these findings also support the notion that the main action of DPP-IV is mediated by an increase of retinal GLP-1 levels.

Nevertheless, as mentioned above, we cannot rule out that other mechanisms unrelated to GLP-1/GLP-1R could be activated. In this regard, it should be noted that G-protein-coupled receptor (GPcR) coagulation factor II receptor-like 1 (F2RL1, previously known as Par2), which is abundant in retinal ganglion cells, can be activated by DPP-IV [27]. After stimulation, F2RL1 promotes angiogenesis and inflammation, and therefore could be an important target when treating diabetic retinopathy. In addition, it has been reported that interleukin-1 receptor antagonist (IL-1RA), a competitive antagonist of IL$1 \beta$ receptors, is a substrate of DPP-IV [28]. Therefore, the inhibition of DPP-IV could mitigate the deleterious role of IL- $1 \beta$ in the pathogenesis of diabetic retinopathy by preventing the cleavage of IL-1RA.

The experimental evidence on the effects of the oral administration of DPP-IVi in diabetic retinopathy is limited and controversial [29-32]. Gonçalves et al [29] showed that sitagliptin prevented changes in the endothelial distribution of tight junction proteins and decreased nitrosative stress, the inflammatory state and cell death by apoptosis in Zucker Diabetic Fatty (ZDF) rats (a rat model of type 2 diabetes). In 
Fig. 6 (a, b) Retinal concentration of glutamate measured by HPLC in the experimental groups. (c, $\mathbf{d})$ Comparison of GLAST

immunofluorescence (red) among representative samples from each experimental group. Nuclei were labelled with Hoechst stain (blue). Scale bars, $10 \mu \mathrm{m}$. Quantification of GLAST immunofluorescence in arbitrary units (AU). $n=10$ mice per group. Results are means $\pm \mathrm{SD}$. (e, f) Western blotting bands of GLAST and densitometric analyses in mouse retinas. $n=4$. $(\mathbf{g}, \mathbf{h})$ Real-time quantitative RT-PCR analysis of GLAST mRNA in mouse retinas. $n=8$. * $p<0.05$ vs $d b / d b$ mice treated with vehicle. Cyp, ciclophyline; GCL, ganglion cell layer; INL, inner nuclear layer; IPL, inner plexiform layer; ONL, outer nuclear layer; OPL, outer plexiform layer
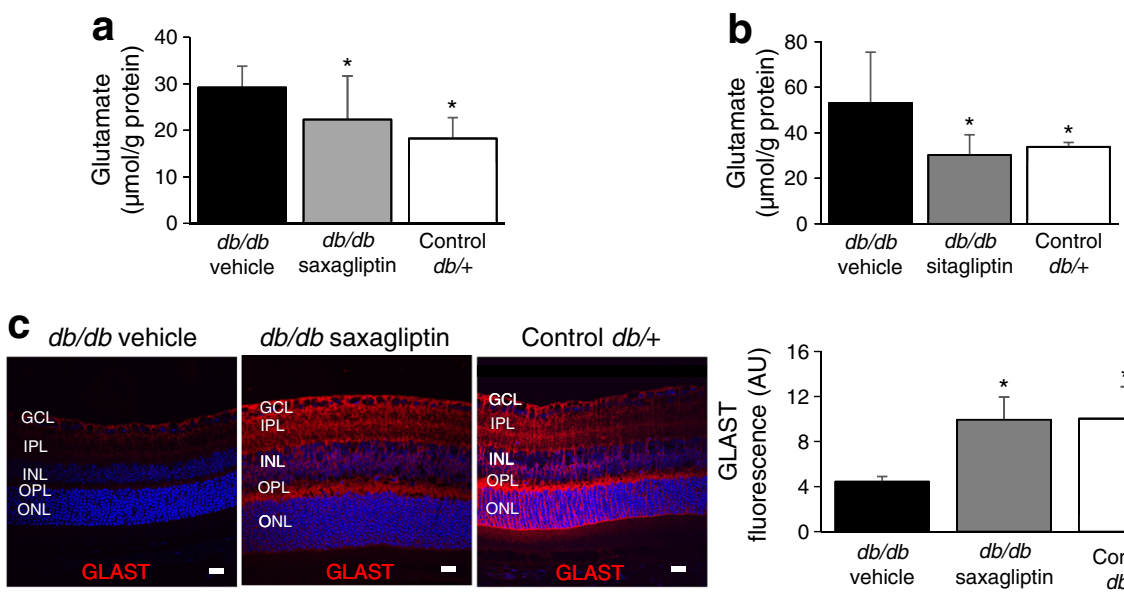

Control $d b /+$
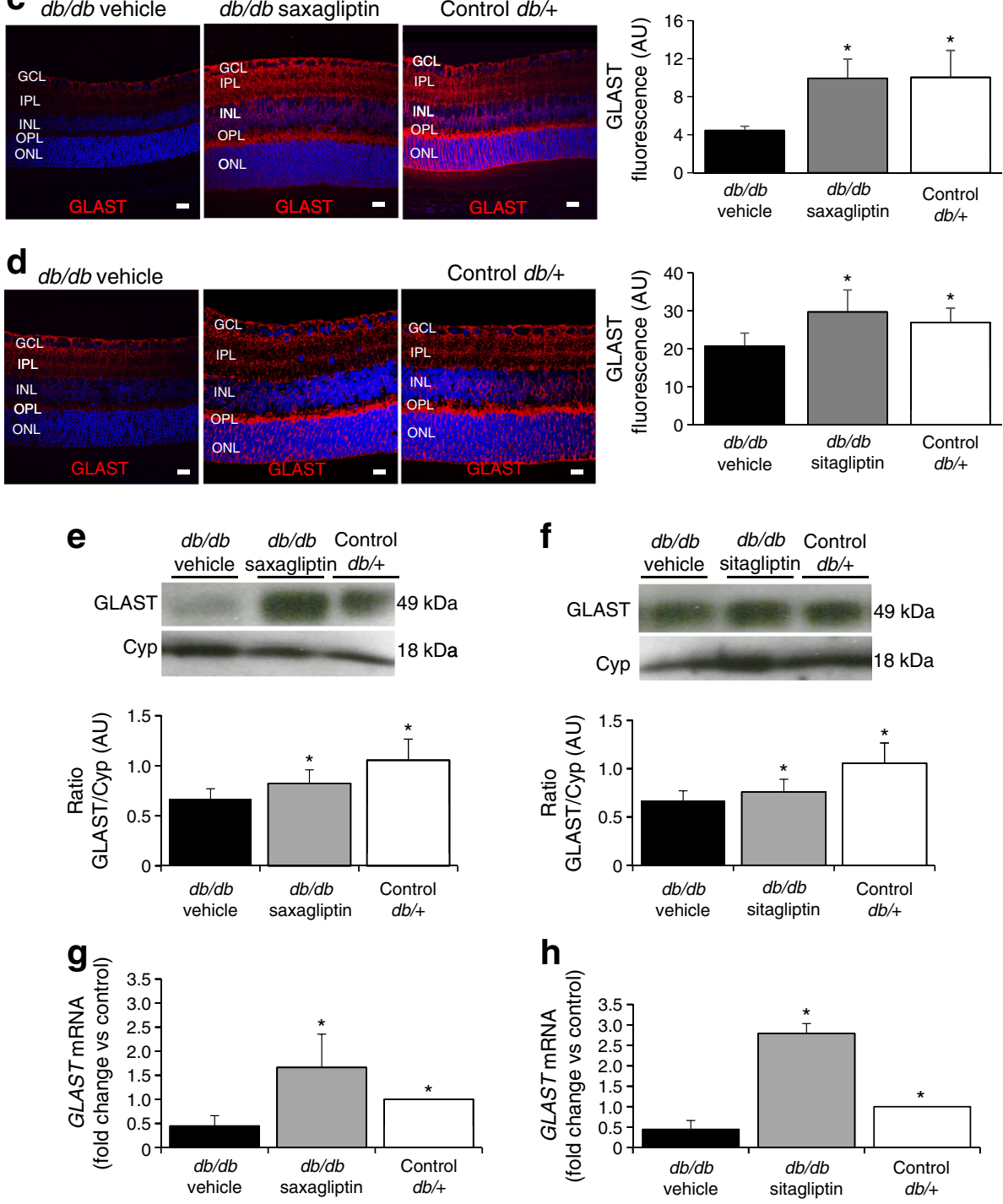

addition, sitagliptin allowed for a recovery of the number of CD $34^{+}$cells present in the bloodstream to levels similar to their number in control animals, while the decreased ability of endothelial progenitor cells (EPCs) to adhere to the retinal vessels that was induced by diabetes was reversed. Even more interestingly, the same authors demonstrated that sitagliptin alleviated the increased BRB permeability in streptozotocin (STZ)-induced diabetic rats, which could not be attributed to a normalisation of glycaemia [31]. However, Lee et al [32] recently reported that the intraperitoneal administration of sitagliptin induced vascular leakage in the retinas of mice with either retinopathy of prematurity or STZ-induced diabetes. The main mechanism involved was the activation of the SDF- $1 \alpha / \mathrm{CXR} 4 / \mathrm{Src} / \mathrm{VE}-$ cadherin signalling pathway. Consequently, further research seems warranted to clarify whether DPP-IVis exert beneficial or deleterious effects in the diabetic retina and also whether this is related to the different routes of administration.

To the best of our knowledge, there has been only one clinical trial specifically addressing the effect of either GLP$1 \mathrm{R}$ agonists or DPP-IV in the eye [33]. In this double-blind, placebo-controlled, crossover clinical trial performed in 50 
Fig. 7 (a) Electroretinogram traces in response to low, medium and high stimulus intensities in a representative non-diabetic mouse (dark blue), a $d b / d b$ mouse treated with vehicle (red) and a $d b / d b$ mouse treated with saxagliptin (green). (b) Quantitative analyses of a-wave and b-wave amplitude in $d b / d b$ mice treated with vehicle (black bars), $d b / d b$ mice treated with saxagliptin (grey bars) and nondiabetic mice (white bars). (c) Electroretinogram traces in a representative non-diabetic mouse (dark blue), a $d b / d b$ mouse treated with vehicle (red) and a $d b / d b$ mouse treated with sitagliptin (green). (d) Quantitative analyses of a-wave and b-wave amplitude in $d b / d b$ mice treated with vehicle (black bars), $d b / d b$ mice treated with sitagliptin (grey bars) and nondiabetic mice (white bars). ${ }^{*} p<0.05$ vs $d b / d b$ mice treated with vehicle
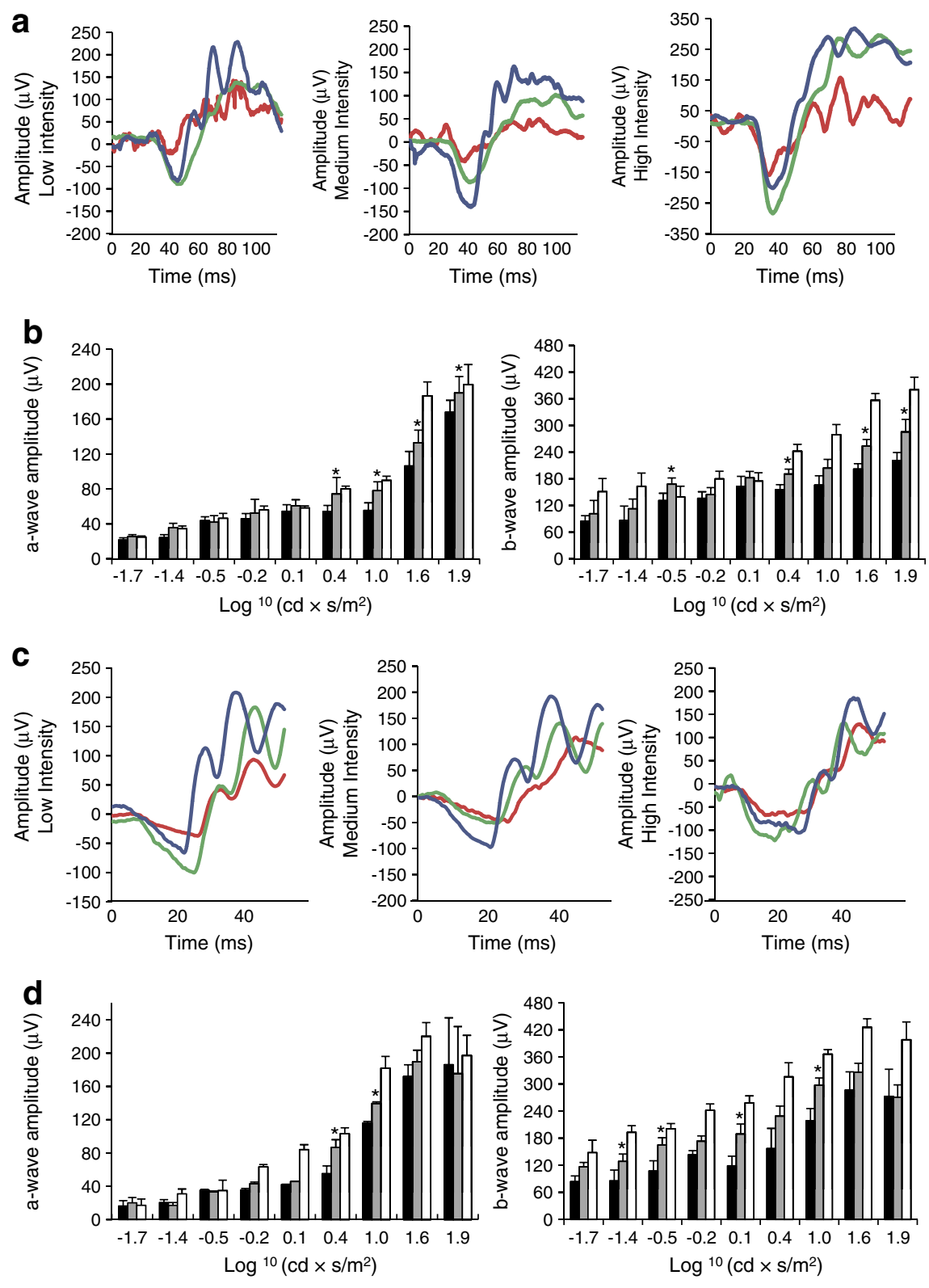

type 2 diabetic individuals without diabetic retinopathy, 6 weeks of sitagliptin treatment normalised retinal capillary flow and increased vasodilatory capacity during flicker-light exposure. However, no specific studies have so far evaluated the effects of GLP-1R or DPP-IVi on the endpoints of diabetic retinopathy.

Recent results of the Cardiovascular and Other Long-term Outcomes with Semaglutide in Subjects with Type 2 Diabetes (SUSTAIN-6) study [34] showed that semaglutide (a longacting GLP-1 analogue with an extended half-life of approximately 1 week) reduced the rate of cardiovascular morbidity and mortality but increased the progression of diabetic retinopathy. This merits comment because it contravenes the experimental results showing the beneficial effects of GLP-1R agonists in the development of diabetic retinopathy [9, 35-40]. In this study, an unexpectedly higher rate of severe complications of diabetic retinopathy (i.e. vitreous haemorrhage, blindness, or conditions requiring treatment with an intravitreal agent or photocoagulation) in the semaglutide arm were detected in comparison with placebo.

However, several important drawbacks should be mentioned. First, the SUSTAIN-6 study was aimed at assessing cardiovascular but not diabetic retinopathy outcomes. In fact, retinal photographs were not taken systematically at baseline, and therefore it is impossible to know whether the two arms (placebo and semaglutide) were matched in terms of the degree of diabetic retinopathy. This is a crucial point because the degree of diabetic retinopathy is the most important factor in 
Fig. 8 (a) Assessment of albumin extravasation by doubleimmunofluorescence labelling (red, albumin; green, collagen IV); magnifications of merged images are shown below. Nuclei were labelled with Hoechst stain (blue). Scale bars, $10 \mu \mathrm{m}$. (b) Confocal immunofluorescence images of vascular permeability assessed by Evans Blue dye (red) leakage in retinal whole mounts. Scale bars, $30 \mu \mathrm{m}$. For quantification, four mice from each group were analysed. $* p<0.05$ vs $d b / d b$ mice treated with vehicle. AU, arbitrary units; GCL, ganglion cell layer; INL, inner nuclear layer; ONL, outer nuclear layer; Spec3, fluorescent spectral signature 3 a

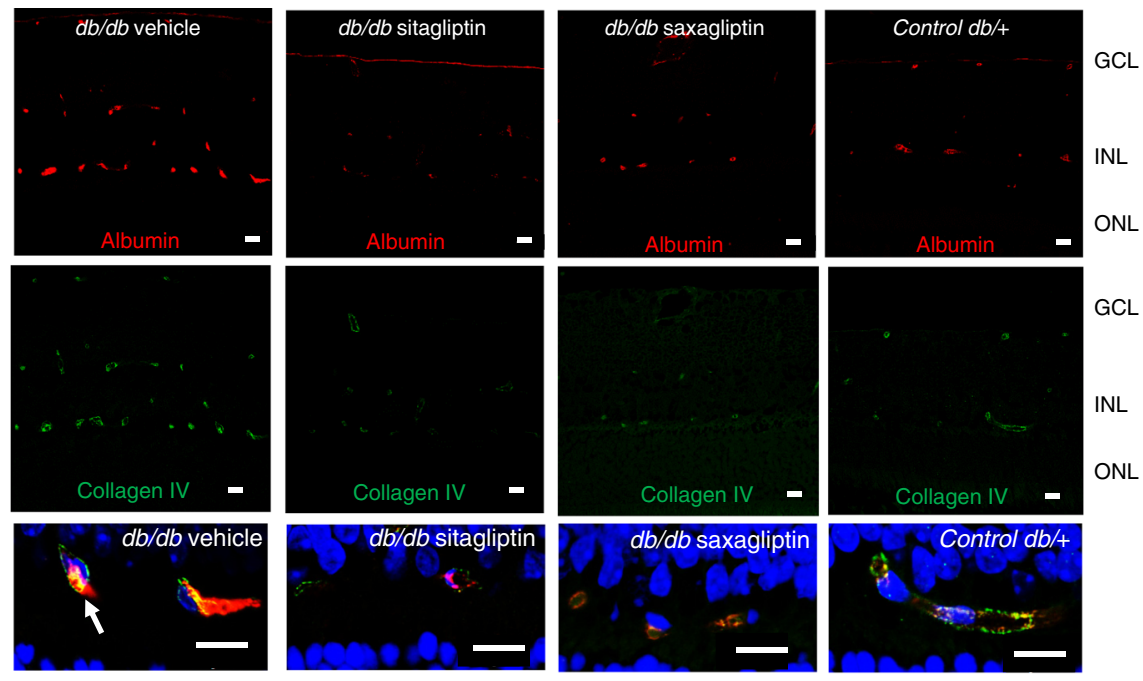

b

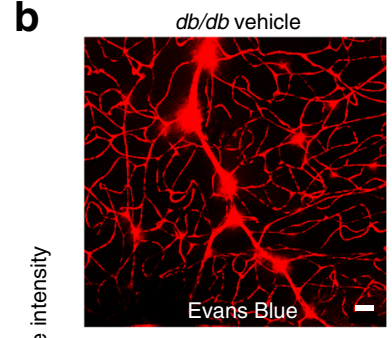

$d b / d b$ sitagliptin
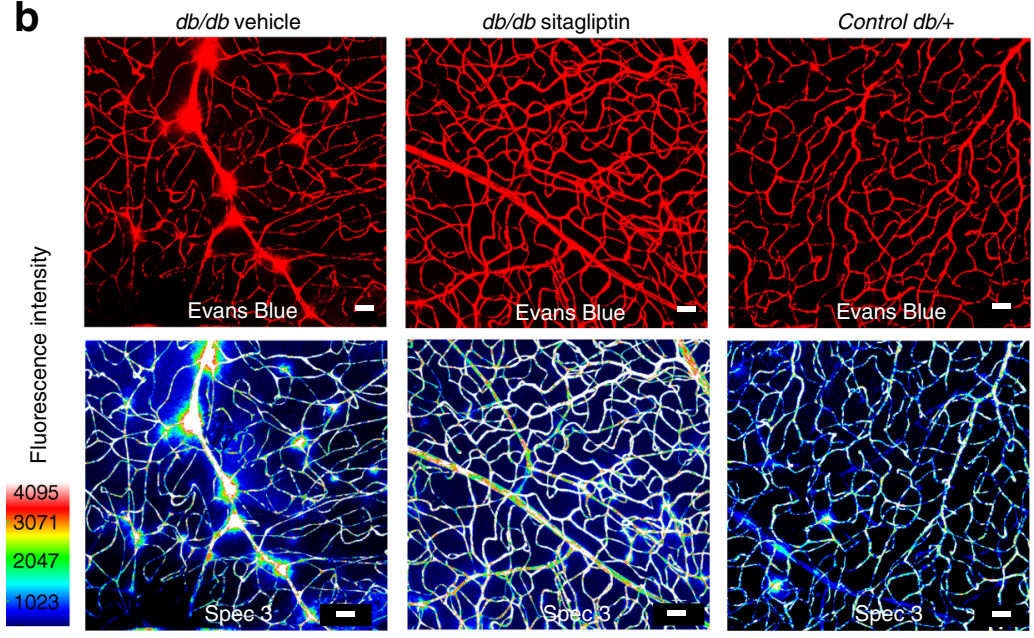

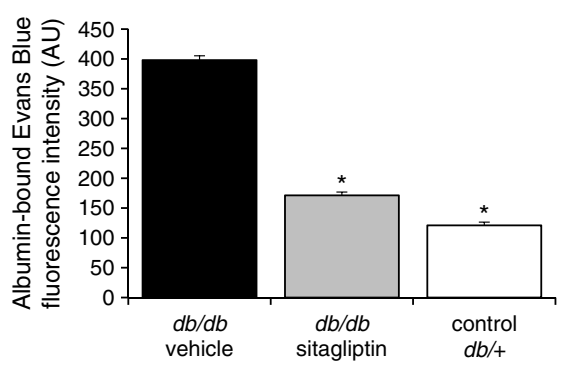

Table 1 Evolution of blood glucose and body weight of the different experimental groups to evaluate the effect of saxagliptin

\begin{tabular}{|c|c|c|c|c|}
\hline & \multicolumn{2}{|c|}{ Blood glucose $(\mathrm{mmol} / \mathrm{l})$} & \multicolumn{2}{|l|}{ Weight (g) } \\
\hline & Baseline & End & Baseline & End \\
\hline$d b / d b$ - vehicle & $30.4 \pm 4.8$ & $32.0 \pm 3.0$ & $45.7 \pm 2.1$ & $47.6 \pm 2.3$ \\
\hline$d b / d b$ - saxagliptin & $29.9 \pm 4.6$ & $31.0 \pm 4.1$ & $46.3 \pm 1.5$ & $49.3 \pm 1.5$ \\
\hline$d b /+$ (control) & $8.8 \pm 1.3$ & $8.8 \pm 1.5$ & $27.5 \pm 1.3$ & $28.4 \pm 2.1$ \\
\hline
\end{tabular}

Data are mean $\pm \mathrm{SD}$
Table 2 Evolution of blood glucose and body weight of the different experimental groups to evaluate the effect of sitagliptin

\begin{tabular}{lrrrrr}
\hline & \multicolumn{2}{c}{ Blood glucose $(\mathrm{mmol} / \mathrm{l})$} & & Weight $(\mathrm{g})$ & \\
\cline { 2 - 3 } \cline { 5 - 6 } \cline { 5 - 6 } & \multicolumn{1}{c}{ Baseline } & \multicolumn{1}{l}{ End } & & Baseline & \multicolumn{1}{l}{ End } \\
\hline$d b / d b-$ vehicle & $28.8 \pm 4.6$ & $30.2 \pm 3.0$ & & $46.3 \pm 2.0$ & $49.5 \pm 1.4$ \\
$d b / d b-$ sitagliptin & $30.2 \pm 3.5$ & $31.6 \pm 2.7$ & & $48.6 \pm 1.4$ & $51.1 \pm 1.6$ \\
$d b /+($ control $)$ & $8.6 \pm 1.0$ & $9.2 \pm 1.3$ & & $28.0 \pm 1.6$ & $28.8 \pm 1.4$ \\
\hline
\end{tabular}

Data are mean $\pm \mathrm{SD}$ 
determining evolution during follow-up. Another potential factor accounting for the results reported on diabetic retinopathy from SUSTAIN-6 is the deleterious effect of a rapid lowering of $\mathrm{HbA} 1 \mathrm{c}$. This could have been a relevant factor regarding individuals in the most severe baseline retinopathy category [41]. However, this critical information was not available due to the absence of diabetic retinopathy grading. A comprehensive review regarding the limiting factors of this study, which prevent valid results for diabetic retinopathy outcomes from being drawn, has recently been published [25].

In conclusion, the topical administration of the DPP-IVi saxagliptin and sitagliptin is effective in preventing neurodegeneration and vascular leakage in the diabetic retina. These effects can be attributed to an enhancement of GLP-1, but other mechanisms unrelated to the prevention of GLP-1 degradation cannot be ruled out. These findings could pave the way for clinical trials testing this new approach, alone or in combination with GLP-1, in the treatment of early stages of diabetic retinopathy.

Data availability All relevant data are included in the article and/or the ESM files.

Funding This study was supported by grants from the Ministerio de Economía y Competitividad (SAF2016-77784 and PI16/00541) and the Fundació Marató TV3.

Duality of interest The authors declare that there is no duality of interest associated with this manuscript.

Contribution statement $\mathrm{CH}, \mathrm{PB}, \mathrm{CS}, \mathrm{JS}, \mathrm{MV}, \mathrm{XG}, \mathrm{OS}, \mathrm{MG}$ and RS made substantial contributions to the conception and design of the study, the acquisition of data, or analysis and interpretation of the data. All authors drafted the article or revised it critically for important intellectual content. All authors approved the final version of the manuscript to be published. RS is the guarantor of this work.

\section{References}

1. Simó R, Hernández C (2015) Novel approaches for treating diabetic retinopathy based on recent pathogenic evidence. Prog Retin Eye Res 48:160-180

2. Stitt AW, Curtis TM, Chen M et al (2016) The progress in understanding and treatment of diabetic retinopathy. Prog Retin Eye Res 51:156-186

3. Simó R, Hernández C, European Consortium for the Early Treatment of Diabetic Retinopathy (EUROCONDOR) (2012) Neurodegeneration is an early event in diabetic retinopathy: therapeutic implications. Br J Ophthalmol 96:1285-1290

4. Antonetti DA, Klein R, Gardner TW (2012) Diabetic retinopathy. N Engl J Med 366:1227-1239

5. Abcouwer SF, Gardner TW (2014) Diabetic retinopathy: loss of neuroretinal adaptation to the diabetic metabolic environment. Ann N Y Acad Sci 1311:174-190

6. Simó R, Hernández C, European Consortium for the Early Treatment of Diabetic Retinopathy (EUROCONDOR) (2014)
Neurodegeneration in the diabetic eye: new insights and therapeutic perspectives. Trends Endocrinol Metab 25:23-33

7. Carrasco E, Hernández C, Miralles A, Huguet P, Farrés J, Simó R (2007) Lower somatostatin expression is an early event in diabetic retinopathy and is associated with retinal neurodegeneration. Diabetes Care 30:2902-2908

8. Garcia-Ramírez M, Hernández C, Villarroel M et al (2009) Interphotoreceptor retinoid-binding protein (IRBP) is downregulated at early stages of diabetic retinopathy. Diabetologia 52:26332641

9. Hernández C, Bogdanov P, Corraliza L et al (2016) Topical administration of GLP-1 receptor agonists prevents retinal neurodegeneration in experimental diabetes. Diabetes 65:172-187

10. Holst JJ (2007) The physiology of glucagon-like peptide 1. Physiol Rev 87:1409-1439

11. Hernández C, García-Ramírez M, Corraliza L et al (2013) Topical administration of somatostatin prevents retinal neurodegeneration in experimental diabetes. Diabetes 62:2569-2578

12. Marmor MF, Holder GE, Seeliger MW, Yamamoto S, International Society for Clinical Electrophysiology of Vision (2004) Standard for clinical electroretinography (2004 update). Doc Ophthalmol 108:107-114

13. Bogdanov P, Corraliza L, Villena JA et al (2014) The $\mathrm{db} / \mathrm{db}$ mouse: a useful model for the study of diabetic retinal neurodegeneration. PLoS One 9:e97302

14. Anderson PJ, Watts H, Hille $\mathrm{C}$ et al (2008) Glial and endothelial blood-retinal barrier responses to amyloid-beta in the neural retina of the rat. Clin Ophthalmol 2:801-816

15. Xu Q, Qaum T, Adamis AP (2001) Sensitive blood-retinal barrier breakdown quantitation using Evans blue. Invest Ophthalmol Vis Sci 42:789-794

16. Xu H, Forrester JV, Liversidge J, Crane I (2003) Leukocyte trafficking in experimental autoimmune uveitis: breakdown of bloodretinal barrier and upregulation of cellular adhesion molecules. J Invest Ophthalmol Vis Sci 44:226-234

17. Anand-Apte B, Ebrahem Q, Cutler A et al (2010) Betacellulin induces increased retinal vascular permeability in mice. PLoS One 5: e13444

18. Schindelin J, Arganda-Carreras I, Frise E et al (2012) Fiji: an opensource platform for biological-image analysis. Nat Methods 9:676-682

19. Narayan SB, Ditewig-Meyers G, Graham KS, Scott R, Bennett MJ (2011) Measurement of plasma amino acids by ultraperformance liquid chromatography. Clin Chem Lab Med 49:1177-1185

20. de Rooij J, Zwartkruis FJ, Verheijen MH et al (1998) Epac is a Rap1 guanine-nucleotide-exchange factor directly activated by cyclic AMP. Nature 396:474-477

21. Kooistra MR, Corada M, Dejana E, Bos JL (2005) Epac1 regulates integrity of endothelial cell junctions through VE-cadherin. FEBS Lett 579:4966-4972

22. Breckler M, Berthouze M, Laurent AC, Crozatier B, Morel E, Lezoualc'h F (2011) Rap-linked cAMP signaling Epac proteins: compartmentation, functioning and disease implications. Cell Signal 23:1257-1266

23. Birukova AA, Tian Y, Dubrovskyi O et al (2012) VE-cadherin trans-interactions modulate Rac activation and enhancement of lung endothelial barrier by iloprost. J Cell Physiol 227:3405-3416

24. Boddu SHS, Gupta H, Patel S (2014) Drug delivery to the back of the eye following topical administration: an update on research and patenting activity. Recent Pat Drug Deliv Formul 8:27-36

25. Simó R, Hernández C (2017) GLP-1 as a target for the treatment of diabetic retinopathy: friend or foe? Diabetes 66:1-8

26. Dietrich N, Kolibabka M, Busch S et al (2016) The DPP4 inhibitor linagliptin protects from experimental diabetic retinopathy. PLoS One 11:e0167853

27. Wronkowitz N, Görgens SW, Romacho T et al (2014) Soluble DPP4 induces inflammation and proliferation of human smooth 
muscle cells via protease-activated receptor 2. Biochim Biophys Acta 1842:1613-1621

28. Zhang H, Maqsudi S, Rainczuk A et al (2015) Identification of novel dipeptidyl peptidase 9 substrates by two-dimensional differential in-gel electrophoresis. FEBS J 282:3737-3757

29. Gonçalves A, Leal E, Paiva A et al (2012) Protective effects of the dipeptidyl peptidase IV inhibitor sitagliptin in the blood-retinal barrier in a type 2 diabetes animal model. Diabetes Obes Metab 14: 454-463

30. Maeda S, Yamagishi S, Matsui T et al (2013) Beneficial effects of vildagliptin on retinal injury in obese type 2 diabetic rats. Ophthalmic Res 50:221-226

31. Gonçalves A, Marques C, Leal E et al (2014) Dipeptidyl peptidaseIV inhibition prevents blood retinal barrier breakdown, inflammation and neuronal cell death in the retina of type 1 diabetic rats. Biochim Biophys Acta 1842:1454-1463

32. Lee CS, Kim YG, Cho HJ et al (2016) Dipeptidyl peptidase-4 inhibitor increases vascular leakage in retina through VE-cadherin phosphorylation. Sci Rep 6:29393

33. Ott C, Raff U, Schmidt S et al (2014) Effects of saxagliptin on early microvascular changes in patients with type 2 diabetes. Cardiovasc Diabetol 13:19

34. Marso SP, Bain SC, Consoli A et al (2016) Semaglutide in cardiovascular outcomes in patients with type 2 diabetes. N Engl J Med 375:1834-1844
35. Zhang Y, Wang Q, Zhang J, Lei X, Xu G-T, Ye W (2009) Protection of exendin-4 analogue in early experimental diabetic retinopathy. Graefes Arch Clin Exp Ophthalmol 247:699-706

36. Zhang Y, Zhang J, Wang Q et al (2011) Intravitreal injection of exendin-4 analogue protects retinal cells in early diabetic rats. Invest Ophthalmol Vis Sci 52:278-285

37. Fu Z, Kuang HY, Hao M, Gao XY, Liu Y, Shao N (2012) Protection of exenatide for retinal ganglion cells with different glucose concentrations. Peptides 37:25-31

38. Fan Y, Liu K, Wang Q, Ruan Y, Zhang Y, Ye W (2014) Exendin-4 protects retinal cells from early diabetes in Goto-Kakizaki rats by increasing the $\mathrm{Bcl}-2 / \mathrm{Bax}$ and $\mathrm{Bcl}-\mathrm{xL} / \mathrm{Bax}$ ratios and reducing reactive gliosis. Mol Vis 10:1557-1568

39. Fan Y, Liu K, Wang Q, Ruan Y, Ye W, Zhang Y (2014) Exendin-4 alleviates retinal vascular leakage by protecting the blood-retinal barrier and reducing retinal vascular permeability in diabetic Goto-Kakizaki rats. Exp Eye Res 127:104-116

40. Zeng Y, Yang K, Wang F et al (2016) The glucagon like peptide 1 analogue, exendin-4, attenuated oxidative stress-induced retinal cell death in early diabetic rats through promoting Sirt1 and Sirt3 expression. Exp Eye Res 151:203-211

41. The Diabetes Control and Complications Trial Research Group (1998) Early worsening of diabetic retinopathy in the Diabetes Control and Complications Trial. Arch Ophthalmol 116:874-886 